\title{
THE CONTINUUM AND THE SECOND NUMBER CLASS
}

\author{
$B y$ G. H. HARDY.
}

[[Received August 3rd, 1905.-Read November 9th, 1905.]

1. In a recent number of these Proceedings* Dr. Hobson criticises (among other things) a construction which I gave in $1903+$ for a set of points of cardinal number $N_{1}$ contained in the linear continuum $(0,1)$. This criticism is merely incidental to a much more comprehensive attack on the whole theory of Cantor's transfinite numbers, as it has been generally accepted by mathematicians, and in particular to the theor of cardinals elaborated by Mr. Whitehead and Mr. Russell, and expounded in the latter's Principles of Mathematics. It is, I believe, Mr. Russell's intention to reply to Dr. Hobson, and I should not wish to discuss the general question in the present communication, even if I felt competent to do so. Iry present object is a much more modest one. Besides the large question with which we are all concerned there is a smaller one mhich concerns only Dr. Hobson and myself. Each of us is of opinion that the other has made a mathematical mistake. It is with this smaller question that I propose to deal now, and I shall only refer to the larger issue in so far as is necessary if $I$ am to make clear what the difference between us really is. If Dr. Hobson's views concerning cardinal numbers in general were correct, my construction would acquire a fundamental importance which I am not myself at all disposed to attach to it: I need therefore make no apology for considering in detail this particular part of Dr. Hobson's paper.

2. Before I proceed to discuss Dr. Hobson's objections to my construction, it will be convenient if $I$ indicate a slight simplification $\ddagger$ which can be made in it. Whether this alteration be made or not in no way affects the force of Dr. Hobson's arguments.

- Proc. London Math. Soc., New Series, Vol. 3, p. 170.

† Qharterly Journal, Vol. exxr., p. 88.

† Suggested by a passage in Baire's Leçons sur les Fonctions disoonstinues, p. 25. 
The principle of my construction was to associate with every number $a$ of the second class an ascending sequence

(a) $\quad a_{1}, a_{2}, a_{9}, \ldots$

of positive integers by means of the three following rules :-

(i.) To the number 1 is to correspond the sequence

$$
1,2,3, \ldots .
$$

(ii.) The sequence for $a+1$ is to be formed by omitting the first term of the sequence for $a$.

(iii.) If $\alpha$ is a number of the second kind (one with no immediate predecessor), we are to select a fundamental sequence $\left(\alpha_{\nabla}\right)$ of which $\alpha$ is the limit, and we are to traverse the array

$$
\begin{aligned}
& \left(a_{1}\right) a_{1,1}, a_{1,2}, a_{1,3}, \ldots \text {, } \\
& \left(a_{2}\right) \quad a_{2,1}, a_{2,2}, a_{2,3}, \ldots \text {, }
\end{aligned}
$$

diagonally, so that we obtain

$$
\text { (a) } a_{1,3}, a_{2,2}, a_{3,3}, \ldots
$$

I found then that in order to assure ourselves that the sequences thus generated are all distinct it may be necessary to substitute for the fundamental sequence $\left(\alpha_{\nu}\right)$ another sequence $\left(a_{\nu}+m_{\nu}\right)$, where the $m_{\nu}$ 's are finite numbers formed successively according to a definite rule which I gave in my former paper.* I now wish to point out that this slight complication is quite unnecessary if, instead of defining the sequence for $a$ by the simple equation

$$
a_{n}=a_{n, n},
$$

we take $a_{n}$ to be the greatest of the integers $a_{1, n}, a_{2, n}, \ldots, a_{n, n}$. It is then easy to prove, without the introduction of the numbers $m_{v}$, that, if $a, \alpha^{\prime}\left(\alpha<\alpha^{\prime}\right)$ are any two numbers of the second class, there is a definite $n$ from and after which $a_{n}^{\prime}>a_{n}$; so that notwo sequences can be the same. For, if this is true for all numbers $\leqslant \alpha^{\prime}$ (say), it is obviously true for all numbers $\leqslant \alpha=a^{\prime}+1$. We have therefore only to show that, if it is true for all numbers $\leqslant \alpha_{\nu}$, where $\alpha_{\nu}$ is an arbitrary member of a fundamental sequence $\left(\alpha_{v}\right)$, it is true for all numbers $\leqslant \alpha$, the number which immediately follows this fundamental sequence. According to the construction, $a_{n}$ is the greatest of

$$
a_{1, n}, a_{2, n}, \ldots, a_{n, n} .
$$


Then, if $\beta<a$, we can find $m$ so that $\beta<a_{m}$, and we can find $n_{0}$ so that $n_{0}>m$ and $a_{m, n}>b_{n}$ for $n \geqslant n_{0}$. Thus

$$
a_{n} \geqslant a_{m, n}>b_{n},
$$

and so the sequences for $\beta$ and for $\alpha$ are distinct.

It follows that, if all the sequences which correspond to numbers $<\alpha$ are distinct, all those which correspond to numbers $\leqslant a$ are distinct, whether $a$ has an immediate predecessor or not; and therefore that all the sequences are distinct.

3. Dr. Hobson argues (p. 187) that this construction must be faulty for the following reason. Given any integer $m$, a least number $a_{m}$, he contends, can be found, such that for all numbers $a>a_{m}$ the second term of the corresponding sequence is greater than $m$. Taking a sequence of numbers

$$
m_{1}<m_{2}<\ldots
$$

and forming the number $a$ which is the limit of the fundamental sequence $\left(\alpha_{m_{u}}\right)$, he deduces that the second term in the sequence for $\alpha$ is greater than $m_{\nu}$, for all values of $\nu$, i.e. is greater than any assignable integer, and therefore that no such sequence exists. And, in fact, he concludes that, if the sequences for the early numbers of the second class are formed as I formed them in my earlier paper, no sequence can be constructed to correspond to the number

where

$$
\epsilon_{\omega}=\lim \epsilon_{v},
$$

$$
\begin{gathered}
\epsilon_{1}=\lim \omega, \omega^{\omega}, \omega^{\omega^{*}}, \ldots, \\
\epsilon_{2}=\lim \epsilon_{1}, \epsilon_{1}^{e_{1}}, \epsilon_{1}^{\epsilon_{1}{ }^{\varepsilon_{1}}}, \ldots, \\
\quad \ldots \quad \ldots \quad \ldots \quad \ldots \\
\epsilon_{\nu+1}=\lim \epsilon_{\nu}, \epsilon_{\nu}^{\epsilon_{\nu}}, \epsilon_{\nu}^{\epsilon_{\nu} e_{\nu}}, \ldots . .^{*}
\end{gathered}
$$

I hardly think that Dr. Hobson can have realised how paradoxical his conclusion is. For it follows from the definition of the construction that, if sequences have been assigned by it for all numbers $\langle a$, a sequence is assigned by it for $a$. Therefore, if there is no sequence for $a$, we can find a number $<a$, say $a^{(1)}$, for which there is no sequence, a number $a^{(2)}<a^{(1)}$ for which there is no sequence, and so on. But, as it is impossible to find an infinite descending sequence $a, a^{(1)}, a^{(2)}, \ldots$, we shall find ultimately that there are no sequences at all; and even Dr. Hobson is not so sceptical as this.

- It is not difficult to prove that the numbers thus defined are the ame the first few of Cantor's e-numbers. 
My answer to Dr. Hobson's argument consists simply of a denial of his major premiss. It is not true that, given any integer $m$, a number $a_{m}$ can be found such that, for every $a>a_{m}, a_{2}>m$. The second terms of our sequences may go on increasing for a while, but sooner or later a sudden jump downwards will occur. This is most obvious, perhaps, if we consider a fundamental sequence of the type $(a+\nu)$.

If for $\alpha$ the second term is $a_{2}$, for $\alpha+\nu$ the second term is $a_{2}+\nu$, and we can find a value of $\nu$ for which this number is greater than any assignable number. But it by no means follows that the second term in the sequence for $\alpha+\omega$ is greater than any assignable number; on the contrary, it is $a_{9}$, the second term in the sequence for $a+1$, and is less than the second terms in the sequences for $a+2, a+3, \ldots$ And, more generally, if $b_{n}$ is the $n$-th term in the sequence for $a+\omega$, we can find a value of $\mu$ such that the $n$-th terms in the sequences for $a+\nu(\nu \geqslant \mu)$ are all $>b_{n}$. It is equally true, as I showed in $\S 2$, that, given $\nu$, we can find $n_{0}$ so that for $n \geqslant n_{0}$ the figures in the sequence for $\alpha+\omega$ are greater than those in the sequence for $a+\nu$. That these two propositions should simultaneously be true may seem paradoxical for a moment, but we have only to consider the sequences

$$
\begin{aligned}
& \text { (1) } 1, \quad 2, \quad 3, \quad 4, \quad \ldots \text {, } \\
& \text { (2) } 2, \quad 3, \quad 4, \quad 5, \quad \ldots \text {, } \\
& \text { (v) } \nu, \nu+1, \nu+2, \nu+3, \ldots \text {, } \\
& \text { (w) } \quad 1, \quad 3, \quad 5, \quad 7, \quad \ldots \text {, }
\end{aligned}
$$

to see that they are in reality perfectly consistent with one another, just as the propositions-
(i.) given $m$, we can find $x_{0}$ so that, for $x \geqslant x_{0}, e^{x}>x^{m}$;
(ii.) given $x_{0}(>1)$, we can find $m_{0}$ so that, for $m \geqslant m_{0}, x_{0}^{m}>e^{x_{0}}$ -are consistent with one another.

It may, no doubt, be the case that for a particular number $m$ (e.g.. $1,000,000)$ all sequences later than an assignable sequence have second figures $>m$. Thus Dr. Hobson asserts that, for every $a \geqslant \omega^{\omega}, a_{2} \geqslant 5$, and this may perfectly well be true. But, if it is true, it will depend on two facts :

(i.) that, for $\alpha=\omega^{\omega}, a_{2} \geqslant 5$;

(ii.) that in constructing the sequences corresponding to higher. 
numbers we never use a limit sequence $\alpha_{1}, \alpha_{2}, \ldots$ containing more than one term $<\omega \omega$.

Whether (ii.) is true or not depends entirely on the particular "norm" chosen for forming the sequences. If I had chosen $1, \omega, \omega^{\omega}, \omega^{\omega^{*}}, \ldots$ instead of $\omega, \omega^{\omega}, \omega^{\omega}, \ldots$ as the fundamental sequence for $\epsilon_{1}$, the second term in the corresponding sequence would have been 3. And what Dr. Hobson's argument really proves is simply that, however the sequences are chosen, what may be true for some particular values of $m$ cannot be true for all values of $m$, and that no such proposition as (ii.) above can be true for more than an enumerable sequence of values of $\alpha$ (such as $\omega^{\omega}$ ), which is otherwise obvious. And where his argument breaks down with regard to the particular sequence of numbers $\left(\epsilon_{1}, \epsilon_{2}, \ldots, \epsilon_{\omega}\right)$ which he considers is in his not having noticed that the second term in the sequence for $\epsilon_{\omega}$ is less than the second term in the sequences for $\epsilon_{3}, \epsilon_{4}, \ldots$ (if, as is natural, we take $\epsilon_{1}, \epsilon_{2}, \ldots$ as the fundamental sequence for $\epsilon_{\omega}$ and grant that, as he asserts, the second term in the sequence for $\epsilon_{\nu}$ continually increases with $\nu$ ).

4. The arguments which Dr. Hobson urges specifically against me appear to me therefore to be invalid. These arguments are (as I think Dr. Hobson and I agree) quite independent of those used by him in his general attack on the theory of transfinite cardinals; and I fully admit that the latter cannot be answered in so summary a manner. These arguments will be discussed in detail by Mr. Russell. I shall only refer to them now in order to make clear the point that Dr. Hobson has made against a great deal of generally accepted mathematical reasoning, of which my construction is an average specimen. The point is this, that a great deal of such reasoning really depends on the acceptance of a certain logical postulate of which no proof has yet been given, namely, the postulate of the existence of the multiplicative class. If we have a class of mutually exclusive classes $k$, no one of which is null, the multiplicative class of the $k$ 's is defined as the class of classes each member of which contains one and only one member of each of the $k$ 's. ${ }^{*}$ The class can always be defined, but it has never been proved that it is never null, that is to say, that it always contains at least one member.

Among mathematical proofs in which the existence of the multiplicative

* A. N. Whitehead, “ On Cardinal Numbers" (American Journal, Vol. xxiv., p. 383). 
class is assumed, if not universally, at any rate in cases more extensive than those for which its existence has been proved, I may instance Bernstein's and König's theorems concerning the exponentiation of cardinals, Bernstein's and my own proofs that the cardinal number of the continuum is greater than or equal to $N_{1}$, and Borel's construction for a function of arbitrary class, ${ }^{*}$ among many others.

The last instance is peculiarly instructive, as it shows how assumptions equivalent to that of the existence of the multiplicative class find their way into the writings even of mathematicians who can recognise the assumption elsewhere.

M. Borel wishes to show that functions exist which cannot be represented as double series of polynomials. $t$ If

$$
P_{a, \beta}(x)=\sum_{\gamma=0}^{a+\beta} c_{a, \beta, \gamma} x^{\gamma}
$$

(he says), every double series of polynomials can be written in the form

$$
\sum_{a=1}^{\infty} \sum_{\beta=1}^{\infty} P_{a, \beta}(x) \text {. }
$$

If this series converges for $0 \leqslant x \leqslant 1$, it represents for those values of $x$. a function of class 0,1 , or 2 ; and every function of class 0,1 , or 2 can be defined in this way by a suitable choice of the constants $c_{a, \beta, \gamma}$. "Chaque fonction est même définie d'une infinité de manières, mais cela n'a pas d'inconvénient pour ce qui suit."

M. Borel's subsequent reasoning depends entirely on one representation of every function of classes 0,1 , and 2 having been selected from among the infinity of representations which correspond to each function, i.e., on the existence of the multiplicative class of the classes formed by all the representations of any given function. Yet M. Borel, criticising Zermelo's article in the Annalen, $\ddagger$ uses language which might have been used by Dr. Hobson: "il me semble que les objections que l'on peut y opposer valent contre tout raisonnement où l'on suppose un choix arbitraire fait une infinité non dénombrable de fois; de tels raisonnements sont en dehors du domaine des mathématiques."

* Leçons sur les fonctions de variables réelles, Note IrI., pp. 156-158.

+ I am not implying any doubt of the correctness of the resuit.

$\ddagger$ Mrath. Ann., Bd. Lx., p. 194 .

$\$$ The aggregate of functions considered above has the cardinal number of the continuum. The logical difficulty is the same whether the choix arbitraire has to be made an enumerable or a non-enumerable infinity of times; but, of course, it will generally be easier. in a particular case in which the infinity of times is enumerable, to get over the difficulty by substituting a " norm," i.e., a set of rules for choosing, for the "arbitrary" acts of choice. 
In the case of my construction the form in which the postulate is used is in the assumption of the existence of the multiplicative class of the class of classes formed by all the progressions whose limit is a given number $a$. To each a corresponds an infinity of such progressions: of these progressions we must select one for every $a$, and it certainly seems very paradoxical to suppose that the class whose members are defined to be all the various aggregates of selected progressions should be null, i.e., possess no members. But, although we can define the class, we cannot (so far as can be seen at present) specify a single one among its members, and there seems to be no way of proving that there are members except by actually producing them.

The instance of the decimals referred to by Dr. Hobson is really not a parallel. For the class of decimals does exist: we can produce some at any rate of its members, for example $0000 \ldots, 0101 \ldots$. Here the multiplicative class is that of an enumerable class of classes each of which contains the tro members 0 and 1 , and its existence can be proved.

The necessary axiom, if it is to be postulated, may be postulated in a variety of forms, that of the assumption of the universal existence of the multiplicative class, or its existence subject to restrictions,* or in either of Zermelo's two forms:

(a) That the product of any number of infinite cardinals cannot be zero.

(b) That a relation exists which correlates each class contained in a given class with one of its members.

It has been proved that $(b)$ implies $(a)$ and the universal existence of the multiplicative class, but whether or no the latter imply the former has not yet been decided. Mr. Russell has traced the consequences of the denial of the multiplicative axiom in the arithmetic of the transfinite numbers, and has shown that the question as to its truth or falsity has no bearing on the question of the Aleph-series and Burali-Forti's contradiction, which must be met in quite another way. There is therefore no reason for supposing that Zermelo's assumption is not valid except that it has not been proved. And that it has not been proved means simply that no general method has been given

- It is, of course, quite possible that the existence of the multiplicative class may not be universal, but may hold in extensive particular cases, e.g., when the class of classes whose multiplicative class is required is well ordered. The multiplicative class certainly exists for any class of well-ordered classes, if a definite order is given with each class, but it has not been proved to exist for a vell-crdered class of any classes. 
for defining in finite terms, i.e., by a finite number of repetitions of a finite number of symbols, one member of the multiplicative class whose existence is to be established. Even if we knew that it was impossible ever to define a single member of a class, it would not of course follow that members of the class did not exist, but there appears to be no way of proving the contrary, except by actually specifying a member or by showing that the hypothesis that there is no member leads to contradiction; and, awkward and paradoxical as the consequences of denying the multiplicative axiom are, it has yet to be shown that they are contradictors.

I am therefore, in default of proof, prepared to accept the multiplicative axiom* provisionally on the grounds

(i.) that to deny it appears to be paradoxical;

(ii.) that no reason has been given for denying it;

(iii.) that to deny it reduces to a state of chaos a great deal of very interesting mathematics.

- I do not imply that I accept Zermelo's proof that every aggregate is well ordered. I agree with Dr. Hobson in thinking it open to objection on other grounds. 